ISSN: $1130-3743$

\title{
CIENCIA Y TECNOLOGÍA: MODOS DE ACERCARNOS A LA REALIDAD EDUCATIVA
}

\author{
Science and technology: ways of getting closer to the \\ educational reality
}

Joan Carles RINCÓN I VERDERA

Universidad de les Islles Balears (UIB). Facultat d'Educació. Departament de

Ciències de l'Educació. Campus Universitari. Carretera de Valldemossa, km 7,5.

07071 Palma de Mallorca (Illes Balears).jrincon@sgtedu.caib.es

Fecha de aceptación definitiva: marzo de 2002

BIBLID [(1130-3743) 13, 2001, 89-113]

RESUMEN

Hoy en día la mayoría de los autores que se dedican a trabajar en el campo de acción propio de la Teoría de la Educación coinciden en destacar que ésta debe ser, al mismo tiempo, científica y tecnológica, es decir, una teoría tecnocientífica. Este trabajo supone una aproximación a los modos epistemológicos de acercarnos e intervenir en la realidad educativa, es decir, una aproximación a los conceptos de ciencia y tecnología, a sus criterios de racionalidad y a la relación que establecen con la educación.

Palabras clave: racionalidad, ciencia, tecnología, ética, epistemología, teoría tecnológica.

\section{SUMMARY}

Nowadays the majority of authors who work in the field of Education Theory coincide in pointing out that it should be both scientific and technological, that is to say a techno-scientific theory. This work is supposed to be an approach to 
epistemological ways of getting closer to and interacting with the educational reality, which implies having to deal with the concepts of science and technology, their criteria of rationality and the relationship they establish with education.

Key words: rationality, science, technology, ethics, epistemology, technological theory.

\section{INTRODUCCIÓN}

Desde una concepción clásica de la ciencia y la tecnología podíamos decir que el conocimiento científico perseguía producir nuevos saberes por medio de la explicación comprensiva de la realidad fáctica (saber por saber); y que el conocimiento tecnológico buscaba producir objetos o alternativas para solucionar problemas prácticos, preocupándose por la funcionalidad práctica de dicho saber, por su utilidad para alcanzar objetivos de acción (saber para hacer). En la actualidad, las diferencias básicas entre ciencia (sistema de conocimientos sobre la realidad) y tecnología (sistema de acciones sobre la realidad), pueden y deben establecerse tomando como referencia las finalidades que persiguen y la racionalidad que se pone en juego en ambas modalidades del saber ${ }^{1}$. Desde esta perspectiva, creo que es posible explicar: primero, que la distancia entre ciencia y tecnología se reduce a una mínima expresión; segundo, que con independencia de las diversas finalidades que fundamentan la racionalidad de la acción educativa, o de la consideración paradigmática y/o metodológica a la que nos adscribamos, podemos llamar tecnológicas a aquellas teorías educativas que con pretensiones pragmáticas (intervenir sobre la realidad educativa para mejorarla), buscan sistematizar ordenadamente las acciones educativas con la finalidad de controlar los procesos educacionales e incrementar la eficacia y efectividad de las actuaciones (anticipar racional y razonablemente el conjunto de acciones a llevar a cabo en nuestra práctica educativa); y, tercero, que la racionalidad tecnológica exige de la racionalidad epistémica y de la racionalidad práctica para el logro de sus objetivos de acción.

\section{Finalidades y Racionalidad en la ciencia y la tecnología}

como ya hemos dicho, desde posicionamientos clásicos se ha entendido que la finalidad básica de la ciencia es la adquisición de conocimientos alejados de intereses prácticos. La ciencia se asocia a la desinteresada búsqueda del conocimiento en sí mismo. Por su parte, la finalidad de la tecnología, que halla para su actuación,

1. Pese a que finalidades, racionalidad y, en definitiva, el propio conocimiento educativo, se hallan afectados por la complejidad del propio contexto en el que se mueven, con lo cual el rigor y la objetividad de la intervención, tanto en la práctica educativa diaria (formal o no formal), como en el campo heurístico o investigacional para mejorar dicha praxis, se mueven en el plano de la incertidumbre. 
a diferencia de la técnica (aplicación de la ciencia), su racionalidad epistémica en la ciencia (reflexión teórica sobre problemas técnicos), se orienta hacia el campo de la acción, es decir, al logro de objetivos prácticos (saber para hacer). Si la ciencia se enmarca en la perspectiva del saber, la tecnología se sitúa en el plano del hacer. No obstante, hoy por hoy, esta dualización de intereses entre el saber y el hacer (Dewey, 1995) parece alejarse de la realidad, pues las finalidades en ambos sistemas son de índole teórico-práctica, de tal modo que se hace difícil introducir esta distinción pues, a menudo, los intereses se superponen y confunden:

[...] la forma técnica más perfeccionada es la que se resuelve en ciencia aplicada; en cambio, la Tecnología aparece cuando el problema de acción que se pretende resolver mediante la aplicación de técnicas es objeto de reflexión teórica; de ahí, entonces, que se pueda afirmar que la Tecnología es la teoría de la técnica, pues la técnica [...es] aplicación de la ciencia [...] El conocimiento no es exclusivo de la aplicación de la mentalidad científica (logro de teorías sobre la realidad) sino también de la que podemos denominar mentalidad tecnológica (logro de acciones sobre la realidad), máxime cuando esta segunda forma de pensar se ha acogido al estatuto teórico (reflexión sobre la técnica) y científico (la técnica no deja de ser ciencia aplicada), de la primera (Colom, 1986, 18).

No nos equivocamos si decimos que tanto la ciencia como la tecnología hallan su identidad y razón de ser en el conocimiento para la acción, y no sólo en el conocimiento por el conocimiento, por lo cual la demarcación o deslinde entre una y otra tan sólo puede llevarse a cabo si tomamos como base diferenciadora la forma o el modus operandi en que ambas llevan a cabo su conocimiento (Liz, 1995).

Distintos autores (Medina Domínguez, 1999; Pestre, 1995; Solís Santos, 1994) destacan que las diferencias establecidas entre el conocimiento teórico de la ciencia (desinteresado) y el conocimiento práctico de la tecnología (interesado) no se dan en la realidad histórica, cuestionando la separación del conocimiento en sus dos acepciones generales: epistéme y téchne, saber y hacer, conocer y aplicar. En la posmodernidad, caracterizada por las nuevas tecnologías de la comunicación y de la información (sociedad del conocimiento), parece dotarse al saber (Toffler, 1991) de una nueva dimensión sobre la base de la informática, la telemática y la robótica, siendo valorado, no sólo en cuanto a sus posibilidades de ser aplicado en la resolución de problemas reales, sino también por sus posibilidades prospectivas (Pagels, 1991; Copeland, 1996; Colom, 1997b; Álvarez Castillo, 2001) y por su capacidad de anticipar probabilísticamente los sucesos venideros mediante los programas de simulación (Miró, 2001). En el campo de lo humano y social y, en particular, en el de la educación, las tecnologías, debido a la gran dificultad para predecir con fiabilidad matemática (Bohm, 1992) las transformaciones y cambios que se producen en estos ámbitos ${ }^{2}$, no pueden evaluar la efectividad de la aplicación del conocimiento

2. La naturaleza viene caracterizada por ser un gran proceso estocástico, donde la nota más definitoria es la impredicibilidad. Lo dicho respecto a la naturaleza puede, perfectamente, ser aplicado a lo social (White, 1990; Bateson, 1993). 
generado en relación con su exactitud científica, sino, como señalaba C. Peñalver (1987), por su pertinencia, es decir, por sus posibilidades de moverse en contextos complejos e inciertos (Wagensberg, 1989; Morin, 1995; Unesco, 1999).

Si aplicamos como criterio de demarcación la manera o forma en la que se operativiza el conocimiento científico y el tecnológico, es mucho más viable apreciar las diferencias que separan ambos tipos de saberes y, al mismo tiempo, comprobar las señas identificativas específicas concretas que dan entidad propia a uno y otro conocimiento (Colom, 1997a). La ciencia opera y se estructura a través de la teoria ${ }^{3}$, en oposición a la tecnología que lo hace a partir del modelo para la predicción de acontecimientos (Balzer, 1997). No obstante, hoy por hoy tales predicciones, son, cuanto menos, complejas porque los modelos no pueden tener en cuenta los estados iniciales ni la interacción total de los elementos de sistemas dinámicos y alejados del estado de equilibrio, moviéndose, por lo tanto, en el espacio de la incertidumbre (Briggs y Peat, 1999; Gleick, 1988). En la ciencia (y las teorías de las que se vale), el conocimiento busca explicar o describir la constitución de la realidad que se trabaja o estudia ${ }^{4}$; en la tecnología (y los modelos de los que se sirve), se busca la producción de objetos o alternativas de solución (Kaufman, 1977) que nos permitan resolver problemas prácticos por medio del diseño y control de los procedimientos que hagan eficaces y eficientes nuestras actuaciones (Castillejo, 1987). Podemos decir que la ciencia nos aporta su capacidad explicativo-descriptiva o, si se quiere, su potencialidad generalizadora (leyes), mientras que los modelos tecnológicos, y con ellos los diferentes diseños de intervención en que éstos se concretan (distintas situaciones o realidades problemáticas particulares), nos aportan su capacidad y funcionalidad para la resolución de los problemas y, por lo tanto, para la toma de decisiones en contextos concretos (normas).

Así pues, las teorías científicas nos muestran los fenómenos tal y cual acontecen en la realidad (o al menos con el máximo rigor posible dada la complejidad estructural de dicha realidad), además de hacernos evidente, con todas las dificultades que ello conlleva, la causalidad subyacente a dicha fenomenología, con la finalidad de poderla conocer adecuada y significativamente (el saber, el conocer o la racionalidad epistémica). En este sentido, creo clarificadoras las palabras de J. D. Novak (1993) cuando nos dice que "la construcción del conocimiento es un producto complejo de la capacidad humana para erigir significados, del contexto cultural y de los cambios evolutivos en las estructuras de conocimiento relevante y

3. Podemos decir, siguiendo a J. L. Hayman (1981), que el grado de desarrollo de una ciencia se halla en relación directa con el interés que muestran los científicos en la elaboración de teorías. Véase, en este mismo sentido, F. N. Kerlinger (1975) y E. López-Barajas (1984).

4. Véase, por ejemplo, el caso de N. Luhmann y su concepción de una teoría sistémica de lo social. Para Luhmann las teorías son constructos apriorísticos, es decir, que no se deducen o infieren de la realidad porque desde el mismo momento en que nos enfrentamos a la realidad necesitamos un aparato teórico para explicarla. En el caso de Luhmann la teoría sociológica es lo que permite describir la realidad social (Luhmann, 1990). 
en los medios de adquisición del nuevo conocimiento". Los modelos, por su parte, nos informarán de la manera en que es más pertinente actuar o intervenir en dicha realidad para satisfacer los objetivos propuestos (el hacer o la racionalidad práctico-instrumental), es decir, la manera en la que debemos articular el conjunto de medios puestos en juego para que la acción resulte eficaz (Sanvisens, 1986; Bunge, 1985a). De todo lo dicho, lo que nos interesa analizar es el tipo de racionalidad con la que ambas actividades humanas (ciencia y tecnología) se corresponden: racionalidad epistémica y racionalidad práctico-instrumental. Esta última, la racionalidad práctico-instrumental, que caracteriza a la tecnologia, ha recibido, sobre todo, desde la Escuela de Francfort (Zamora, 2001) y en la actualidad desde el nuevo sector sociocrítico (Ayuste y otros, 1999), duras críticas derivadas, básicamente, de olvidar la vertiente ético-moral (práctica) que toda acción tecnológica debe suponer, reduciéndola a su concepción instrumental.

\section{CiENCIA Y TECNOLOGÍA: LA INEXISTENTE SEPARACIÓN DE LA RACIONALIDAD EPISTÉMICA Y LA RACIONALIDAD INSTRUMENTAL}

Podemos decir, de acuerdo con la tradicional división de los saberes y del tipo de racionalidad mediante la cual dicho saber se estructura, que la racionalidad epistémica es la propia de la ciencia, mientras que la racionalidad práctico-instrumental lo es de la tecnología. La racionalidad epistémica, propia del conocimiento científico, no requiere de la racionalidad instrumental para fundamentar la validez o viabilidad de sus presupuestos; por su parte, la racionalidad práctica o instrumental, propia de la tecnología, debe, necesariamente, referirse a la racionalidad epistémica, constituyendo este hecho uno de los ejes de distinción y demarcación entre la tecnología (teoría de la técnica) y la técnica (aplicación de la ciencia). Efectivamente, la distinción entre técnica y tecnología radica en que la primera, la técnica, se constituye en un saber cómo actuar sin preocuparse por la reflexión teórica sobre los problemas que quiere resolver; la segunda, la tecnología, por el contrario, quiere saber y saber hacer, es decir, saber por qué y saber cómo (además, no hemos de perder de vista que también quiere saber para qué, es decir, fines y valores), con lo cual proporciona un saber que va mucho más allá de lo simplemente empírico, es decir, proporciona un conocimiento racional sobre los problemas que quiere resolver en consonancia con los criterios pragmáticos de eficacia y utilidad (máxima operatividad de la acción para el logro de los objetivos propuestos con el mínimo coste de recursos y medios empleados para ello).

Lo cierto, sin embargo, es que esta distinción (racionalidad epistémica versus racionalidad práctico-instrumental) obvia, por una parte, el hecho de que todo conocimiento (sea éste científico o tecnológico) remite, en última instancia, a un sistema simbólico de ideas, creencias, preconcepciones, opiniones, valores, símbolos, conceptos, teorías, etc. (García Carrasco, 1986), por lo que en la práctica, la racionalidad instrumental remite, a su vez, no a un objeto, sino a un sujeto o colectivo 
de sujetos que viven inmersos en dicho sistema simbólico-cultural y que, consecuentemente, son capaces, no sólo de representar conceptualmente la realidad, sino también de reflexionar significativamente sobre ella (Gergen, 1996), por lo cual este tipo de racionalidad asumiría, de hecho, la propia racionalidad epistémica (Martínez Miguélez, 1993; Newton-Smith, 2000)5. Si hemos dicho que la racionalidad práctico-instrumental nos remite a la racionalidad epistémica, esta última, paralelamente, también remite a la racionalidad tecnológica, ya que el conocimiento teórico de la realidad también posee una dimensión práctica al incidir directamente en la visión que el sujeto tiene de la realidad y, por consiguiente, en sus posibilidades de actuar en ella (modificándola, cambiándola, transformándola).

La teoría del conocimiento no puede entenderse separada del sistema tecnológico, pues toda separación supondría una escisión injustificada, ya que queremos conocer con el objeto de mejorar la realidad, no por el placer de conocerla. Uno de los criterios de racionalidad en los que se apoyan los sistemas tecnológicos para su estructuración es, precisamente, el criterio de innovación-cambio-movimientodinamicidad y, con él, el criterio de capacidad de control de la realidad con la finalidad de conducir los cambios en la dirección deseada. Este criterio, por lo tanto, no se diferencia mucho del que utiliza la ciencia para determinar su racionalidad, pues la racionalidad epistémica no consiste en acumular por acumular conocimientos, sino en modificar y mejorar dichos conocimientos para ser útiles, para mejorar la propia realidad, con lo cual el acercamiento entre la ciencia y la tecnología para innovar e intervenir en la realidad es más que evidente. Por otra parte, tal distinción parece obviar también que la racionalidad si quiere ser razonable debe remitirse a fines, y que la tecnología, en tanto que conjunto de actuaciones intencionales para la resolución de problemas, halla también en la teleología, la axiología, la moral y la ética las bases para su constitución (Rescher, 1999; Agazzi, 1996)6. En este sentido, E. Laszlo (1990) nos dice que las bifurcaciones brindan a la gente la oportunidad de alterar las probabilidades en el caprichoso juego de las fluctuaciones, con lo cual cada uno de nosotros tiene la posibilidad de construir una nueva era según sus valores y sus ideales.

5. Por otra parte, la actual complejidad de nuestra realidad social (efecto directo, entre otras cosas, del impacto de las nuevas tecnologias de la información) afecta, especialmente, al campo cle las ciencias humanas y sociales que se ven muy limitaclas a la hora de explicar la innovación, el cambio y el movimiento (desaparece la linealidad acumulativa de la causa-efecto). Epistemológicamente, en el campo de las ciencias de la educación, y más concretamente en el de la Teoría de la Educación, se debería dar cuenta de la dinamicidad que caracteriza todo lo educativo. Véase, en este sentido, G. Balandier (1996)

6. La racionalidad de la tecnología debe ser, si no quiere reducirse a su instrumentalidad, subsidiaria o, mejor dicho, debe siempre remitir, no sólo a la racionalidad epistémica, sino también, como nos recuerda el profesor J. L. Aranguren (1982) a la racionalidad prudencial. Cuando se ignora esta premisa es cuando son válidas las críticas de aquellos autores que indican que la racionalidad de la tecnología se reduce a una racionalidad ideológica-político-social, restringiéndose la racionalidad de la tecnología a su acepción meramente instrumental, cuando ésta debe ser práctico-instrumental (Moreno Cádiz, 1989). 
Como vemos, el supuesto abismo entre la racionalidad epistémica y la racionalidad instrumental es más aparente que real, pues ambos constituyen dos niveles de una actividad sistémica mucho más abarcadora, general y global. Por otra parte, pretender en la actualidad conocer la realidad, sobre todo para las ciencias humanas y sociales y, entre ellas, las ciencias de la educación, con carácter universal y absoluto es más un mito que una posibilidad real, tornándose el conocimiento en concreto y relativo (Giordan y Vecchi, 1988). Hoy se tiende a buscar conocimientos sobre contextos específicos y en función de problemas concretos, con lo cual, como ya hemos dicho, el conocimiento científico se dirige a la resolución de problemas prácticos, constitutivos del sistema tecnológico, y no a conocimientos teóricos puros (saber por saber). El conocimiento científico, por lo tanto, se configura como conocimiento limitado, parcial, contextualizado, regionalizado, relativo (Atlan, 1991) y, fundamentalmente, tecnológico al excluir, o al menos minimizar, la búsqueda del saber en sí mismo y, por el contrario, buscar su racionalidad tecno-teleológico-epistémica en el saber para bacer, o dicho en otras palabras, aceptar los límites y las limitaciones de la razón: “[...] la verdadera actitud racional consiste en reconocer los límites de la razón en vez de tratar de cubrir con ella lo que, a lo sumo, puede ser razonable, pero no racional en el sentido científico" (Aranguren, 1983, 111). Así pues, hoy por hoy, en la actual concepción de la ciencia, resulta difícilmente justificable separar tanto el pensamiento de la acción, como la acción del pensamiento (Fox y Fox, 1995).

\section{CRiterios de RACionalidad en la CIENCIA Y EN LA teCNOlogía}

Uno de los criterios sobre los que se evalúa la racionalidad y la cientificidad de los resultados de la investigación científica se basa en el rigor (mejor que exactitud) con que se ejercita el control experimental de las investigaciones desarrolladas por la comunidad científica. Los criterios de cientificidad han sido interpretados de diferente manera por distintos epistemólogos y filósofos de la ciencia pertenecientes a diversos enfoques y corrientes de pensamiento. Efectivamente, cada escuela filosófica ha subrayado diferentes criterios en función de las propiedades de los fenómenos que pretendian conocer. Tradicionalmente se han dado dos orientaciones epistemológicas de carácter filosófico: la racional y la empirista. En cierto modo podríamos aplicar la epistemología racional al conocimiento formal de la educación, y la epistemología empirista a la tecnología educativa. Es evidente que ambos métodos deben combinarse pues, por una parte, la observación y la experiencia precisan del ejercicio racional, y por otra, la reflexión racional necesita sustentarse en una base real para no entrar en un círculo vicioso, perdiéndose en la mera palabrería o en la simple especulación filosófica. El empirismo y el positivismo por él representado (Círculo de Viena), se apoyan en la verificación como criterio de legitimación científica de una teoría, así como por el desarrollo de una metodología experimental que aspira a la descripción de los hechos a partir de la 
formulación nomológica de leyes (Porta, 1983). Estos epistemólogos sólo admiten dos clases de proposiciones, las formales que son tautológicas (lógica y matemática), y las fácticas que son empíricamente verificables. Según esto el resto de proposiciones sólo tienen un carácter ético o emotivo y, consecuentemente, carecen de cientificidad (Rubio, 1984). Esta visión del conocimiento científico como empresa empírico-inductiva, racional y libre de valores fue seriamente cuestionada durante toda la segunda mitad del siglo xx por filósofos de la ciencia como K. Popper, T. S. Kuhn, P. K. Feyerabend o I. Lakatos.

Desde el momento en que no todos los fenómenos son susceptibles de verificación empírica, el criterio de verificabilidad no puede constituirse como universal o absoluto. Al empirismo inductivo, y frente al tradicional criterio positivista de la infalibilidad de la ciencia, el racionalismo crítico propone la falsabilidad como criterio de legitimación científica de una teoría. K. Popper $(1973,1983,1988)$ se desvincula, por lo tanto, de los procesos inductivos en la elaboración de teorías científicas. Para Popper ningún conocimiento procede de la inducción, sino que se origina en un número finito de expectativas e intereses que se van ampliando y definiendo como respuesta a su cumplimiento o incumplimiento, en un proceso de conjetura y refutación similar a la selección natural (Popper, 1988). Ante la falta de fundamento lógico de la verificación empírica, Popper plantea y propone como criterio de cientificidad de una hipótesis o teoría, la alternativa falsacionista, según la cual la validez de éstas vendría asegurada en la medida en que no hubiera podido ser refutada o falseada, y en la medida en que el científico (y esto es muy importante, pues se trata de una actitud o predisposición positiva hacia el avance de la ciencia), tuviera la firme voluntad de poner a prueba sus presupuestos. Así pues, las únicas proposiciones científicas serían aquellas que pueden ser sometidas a falsación mediante el contraste de sus predicciones. La falsabilidad implica, necesariamente, la provisionalidad de la ciencia (la ciencia como sistema abierto, en permanente revisión y construcción), ya que en determinadas circunstancias, contextos o situaciones puede ser que los enunciados observacionales propuestos no den una respuesta adecuada del fenómeno que estamos estudiando o investigando (basta que se presente un único caso que falsee la hipótesis), lo cual implica abandonar la línea de trabajo y buscar nuevas vías de solución (Popper, 1973).

De acuerdo con la epistemología evolucionista que caracteriza la teoría popperiana, el conocimiento humano progresa mediante la elaboración de modelos predictivos (conjetura), su contraste con la realidad (refutación) y la revisión de aquellos que se invaliden. El error popperiano, frente a la verificación a posteriom positivista, es condición necesaria para el avance de la ciencia (una ciencia libre de toda forma dogmática que aprende de sus propios errores), siempre que aquél sea explicitado y debidamente superado (Astolfi, 1999). Desde esta perspectiva, la meta de todo científico debería ser la formulación de teorías falseables, su contraste inmediato con la realidad y su rechazo si no se cumple la predicción. La bondad de una teoría está en función de su falsabilidad; cuanto más teorías rechaza, mayor es su contenido de cientificidad. La filosofía de la ciencia de Popper nos obliga, por 
lo tanto, a considerar todo aquello que damos por válido como simples conjeturas no falseadas de momento, pero abiertas a refutación y eventualmente deshechables. En definitiva, para Popper la ciencia avanza cuando se buscan razones que desconfirmen las propias teorías, lo cual permite no cosificar el segundo mundo popperiano, el mundo de las teorías y de la ciencia (Suppe, 1990). La realidad, sin embargo, es que ambos criterios, verificabilidad y falsación, pueden ser perfectamente compatibles a la hora de validar las teorías científicas pues la verificación otorga solidez al conocimiento científico y la falsación posibilita el avance de la ciencia. Verificación y falsación son aplicables también a la tecnología educativa, si bien con cierto relativismo por lo que respecta a la normativización. La verificación de las normas otorga seguridad al proceso educativo, mientras que la falsación posibilita la mejora de los procesos de intervención.

Por su parte, T. S. Kuhn (1981) se aleja de los planteamientos popperianos, pues ningún proceso histórico descubierto hasta la fecha a través del estudio del desarrollo científico se asemeja al estereotipo metodológico de la demostración de la falsedad por medio de la comparación directa con la naturaleza. Al contrario, es precisamente lo incompleto y lo imperfecto del ajuste entre teoría y datos lo que define muchos de los enigmas que caracterizan a la ciencia normal. Si todos y cada uno de los fracasos en el ajuste sirvieran de base para rechazar las teorías, todas ellas deberían ser rechazadas inmediatamente, con lo cual, como es evidente, acabaría por detenerse la actividad científica. En este sentido, T. S. Kuhn introducirá un nuevo enfoque, defendiendo con P. K. Feyerabend (1999), la inconmensurabilidad de las teorías científicas y, con ello, afirmando la imposibilidad de delimitar criterios objetivos para evaluar la cientificidad de las teorías científicas (Fernández Sáez, 1993). Kuhn relativiza el valor y desarrollo de la ciencia. Frente a la noción de ciencia como sistema teórico unificado, acumulativo, que procede por conjeturas y refutaciones, Kuhn opone la noción de paradigma (Rubio Carracedo, 1984), o lo que es lo mismo, un contexto general en el que se desarrollan las actividades científicas.

Kuhn distingue dos períodos radicalmente diferentes en el desarrollo del conocimiento científico: por una parte, la etapa de ciencia normal; y, por otra, la de la revolución científica (Kuhn, 1995). La primera consiste en la articulación del paradigma con el que se compromete la comunidad, definido como la red de convenciones conceptuales, teóricas, instrumentales y metodológicas que son aceptadas por la ciencia madura. Antes de ese consenso no puede hablarse, al menos con propiedad, de ciencia, ya que no hay acuerdo sobre los temas a investigar ni de cómo hacerlo. Según Kuhn, un paradigma es un logro o realización científica fundamental que incluye, al mismo tiempo, una teoría y algunas aplicaciones ejemplares a los resultados del experimento y de la observación. Se trata de una realización cuyo término queda abierto, que deja aún por hacer toda clase de investigaciones. Además, es una realización aceptada en el sentido de ser recibida por un grupo cuyos miembros no intentan ya rivalizar con ella ni crearle alternativas. Por otra parte, y aquí radica la gran diferencia con Popper, el ajuste entre paradigma 
y realidad no es nunca exacto, y es esa inexactitud la que genera enigmas (puzzles) susceptibles de investigación. Durante su vida profesional, la mayoría de científicos se dedican a la resolución de enigmas que tienen como finalidad dotar a la teoría de un cuerpo de hipótesis auxiliares que permitan explicar los fenómenos con mayor precisión (Chalmers, 1989). Si el positivismo y el racionalismo crítico se ocuparon de la dimensión práctica de la actividad científica y, con ella, de la determinación de criterios objetivos y universales para conformar y confirmar la validez de las teorías científicas, lo paradigmático atiende y responde al nivel histórico-simbólico-cultural y antropológico-axiológico-teleológico de dicha actividad. Precisamente por ello, el paradigma se erige en las señas identificativas de un determinado modo de operar y actuar en la ciencia, es decir, es el principio o marco inspirador (histórico, ideológico, simbólico, cultural, científico, etc.) al que una determinada comunidad científica se somete al optar por la línea de trabajo investigador a seguir.

Ahora bien, cuando la teoría no consigue explicar un número creciente de enigmas, o cuando las hipótesis auxiliares son tantas que hacen confusa y hasta incoherente la teoría, entonces se abre un período incierto en el que se buscan alternativas, pudiéndose proponer un nuevo y alternativo paradigma. El nuevo paradigma no es una modificación del anterior, sino una verdadera revolución científica, es decir, una visión del conocimiento radicalmente diferente. No se trata de buscar nuevas respuestas a las viejas preguntas del paradigma en crisis, sino que, en tanto que revolucionario, lo que hace es formular cuestiones nunca antes planteadas. La transición del viejo al nuevo paradigma, según Kuhn, se produce instantáneamente o, de lo contrario, no llega a ocurrir nunca. A partir de la conversión de un grupo creciente de científicos a los nuevos postulados, el paradigma emergente va ganando poder en el seno de la comunidad científica. El resultado final es inevitable y acaba con el total rechazo del anterior paradigma. El término paradigma, como podemos observar, es un concepto mucho más amplio que el de teoría, pues presupone una determinada visión del mundo y de la realidad, del conjunto de aspiraciones, sentimientos, concepciones e ideas que definen al hombre en un determinado espacio y tiempo, y en el que se integrarán las teorías científicas que respondan a dichas expectativas. El desarrollo y avance de la ciencia no sería otra cosa que el producto del cambio, de la revolución paradigmática, que daría lugar a un nuevo tiempo científico ${ }^{7}$; se trata, por lo tanto, de un nuevo paradigma incompatible con el anterior, de una total remodelación del sistema de creencias vigente ${ }^{8}$. Por otra parte, un paradigma no puede compararse con otro con la finalidad de probar sus méritos relativos, pues ambos parten de postulados

7. En este sentido, respecto a los diferentes tiempos educacionales, véase el excelente trabajo de la profesora C. Romero (2000).

8. La epistemología de los programas de investigación de Lakatos es similar a la de los paradigmas de Kuhn. Un programa de investigación tiene un núcleo estable de hipótesis y principios aceptados como irrefutables y una serie de principios heurísticos que sirven para dirigir la investigación. El 
y presupuestos fundamentales diferentes, dependiendo de la perspectiva adoptada por la comunidad científica, lo cual nos indica que los hechos estudiados y la manera de abordarlos estará en función de la perspectiva propia del paradigma en el que nos coloquemos (Pérez Ransaz, 1999).

De la teoría paradigmática de Kuhn debemos recoger dos puntos básicos: por una parte, que el cambio de paradigma no garantiza un progreso acumulativo, sino discontinuo, con lo cual no es posible asegurar un progreso objetivo, ya que los paradigmas son incomparables; por otra parte, en la formalización del paradigma inciden numerosos elementos de índole social, cultural, política y económica, con lo cual la objetividad estará cargada de subjetividad. Si estos dos puntos inciden poderosamente en la formalización de las teorías de la educación (de las pedagogías), no deberían mostrar tanto su influencia en la Teoría de la Educación, pues ésta contribuye a la creación de estos paradigmas y a dotarlos de cientificidad, ya que los paradigmas educativos son muy vulnerables a las incidencias y agresiones de las ideologías, de las utopías y de las filosofías. Como podemos observar, desde esta perspectiva la ciencia es relativa, pues depende de la historia y la cultura en un tiempo y en un espacio concreto. Las teorías correctas serán las que sean compatibles y coherentes con los significados que se desprenden del correspondiente paradigma, espacio en el que cobran verdadero sentido. Así pues, según Kuhn la coberencia con los significados paradigmáticos es el criterio para determinar la racionalidad o, mejor dicho, es la condición necesaria para aceptar la bondad de una determinada teoría. Lo científico o la cientificidad de una teoría equivaldría a la absoluta sumisión (convicción, creencia... fidelidad casi mágico-religiosa) a los presupuestos del paradigma orientador. Desde estos presupuestos, la ciencia no sería tan sólo de orden lógico, sino también axiológico e ideológico 9 . Neutralidad y objetividad en la ciencia quedan en entredicho. La ciencia es un producto cultural más del hombre (uno entre tantos otros), sin que pueda otorgársele un estatuto diferente o privilegiado, pues se trata de un saber, de un conocimiento más al lado de otros saberes y conocimientos (Horgan, 1998).

mantenimiento de este núcleo estable se realiza mediante readaptaciones del resto del programa, lo que explica la permanencia de lo que Kuhn denomina ciencia normal. La ciencia progresa no sólo mediante el desarrollo de los programas, sino también mediante la sustitución de unos programas por otros. Un programa estará estancado si no logra realizar los descubrimientos que realizan los programas rivales (Lakatos, 1978, 1981; Lakatos y Musgrave, 1970).

9. M. Á. Quintanilla, al exponer la epistemología de los programas de investigación de Lakatos, nos dice que: «[... ] las ideologías (entendidas como formas de pensamiento práctico y totalizador) son imprescindibles en los programas de investigación científica. Recuérdese que entendemos un programa de investigación como un proyecto de intervención en la realidad, proyecto orientado a fines, en el que es inevitable la presencia de un núcleo estable, considerado como irrefutable, así como la de una heurística o conjunto de estrategias, normas y valoraciones que orientan el proceso. Está claro que, de acuerdo con nuestra definición de ideología, estos componentes de todo programa de investigación son ideología en su mayor parte" (Quintanilla, 1978, 110). 
Hoy la ciencia ya no puede ser concebida como una simple y exclusiva actividad teórico-simbólico-verbal, sino eminentemente práctica, funcional, útil, pragmática e intervencionista. La legitimación de la ciencia moderna es, básicamente, tecnológica y no sólo, y exclusivamente, teórico-epistémica, su fundamentación no es el verbo, sino la acción (Hottois, 1999). Como ya he dicho, la ciencia no puede concebirse independientemente del contexto tecnológico al que se vincula. Los posicionamientos vistos se centran exclusivamente en el plano teórico, desvinculándolo de la acción práctica. Lo científico y lo tecnológico (lo tecnocientífico) representan las dos caras de una misma moneda, dos formas complementarias que se reclaman mutuamente para conocer e intervenir en la realidad. En los nuevos tiempos el conocimiento científico se decanta por una orientación hacia la praxis (sin olvidar lo teórico, pues es imposible); y el conocimiento tecnológico, al mismo tiempo, lo hace hacia lo teórico, hacia la reflexión teórica sobre los problemas que desea solventar (sin olvidar lo práctico, pues también es imposible). La ciencia, en definitiva, debe enfocarse hacia la praxis; y la tecnología, por su parte, precisa, si no quiere caer en arbitrariedades, mecanicismos o rutinas, del conocimiento que le aporta la ciencia (Gimeno, 1981). De partida, a la racionalidad tecnológica podemos definirla por los tres tipos de elementos que la configuran: primero, lo epistémico, propio de la ciencia, ya que la tecnología es un saber hacer (el saber por qué), una reflexión teórica sobre los problemas que la técnica quiere resolver; reflexión teórica que debe ofrecer, de forma racional y razonada, explicaciones sobre los procedimientos y actuaciones llevados a cabo; segundo, lo técnico, al estar vinculado a la producción (al hacer en cuanto aplicación práctica de la ciencia), que ofrece la explicación sobre los medios y condiciones considerados más eficientes para el logro de los objetivos propuestos a la acción (el saber cómo); y, tercero, lo ético, al no poder separarse las actuaciones de los grandes principios y fines que les dan sentido (el saber para qué).

Si la racionalidad tecnológica exige de la racionalidad científica, los criterios para evaluar lo razonable de una actuación tecnológica deben ser los mismos que los que se demandan a la ciencia y a la tecnología: por una parte, la utilización de los pasos formales del método científico para acercarnos a la verdad; y, por otra, la eficiencia y la controlabilidad propia de la tecnología en la normativización de los procedimientos. Ahora bien, esta visión es incompleta, pues la acción tecnológica (que siempre es intencional para la consecución de objetivos de valor $)^{10}$, debe también incluir el elemento ético-moral, es decir, debe contemplar, como ya habíamos anticipado, la racionalidad teleológica o, si se me permite, siguiendo una terminología arangureniana, prudencial (Aranguren, 1973, 1982, 1983), ya que la acción tecnológica, desde el momento en que se orienta hacía el logro de objetivos de valor, necesita de la

10. La Teoría de la Educación "[...] deberá trabajar en dos frentes distintos aunque íntimamente relacionados. Por una parte se afanará por entender cómo se hace el hombre y, por otra, su esfuerzo se dirigirá a idear y planificar medios para hacer intencionalmente al hombre” (Puig, 1986, 23). 
deliberación moral, del desarrollo y aplicación del juicio moral a la consideración de los fines de la acción. En este sentido nos adscribimos a la postura defendida por el profesor J. Escámez, cuando nos dice:

[...] Desde nuestra perspectiva la constatación de que la pedagogía haya dependido de cierta filosofía, con consecuencias irrelevantes, no implica que sea innecesario relacionar la pedagogía con la antropología y la axiología. Lo que postulamos es una imagen del hombre desdogmatizada, construida, y constantemente revisada, con los datos de las ciencias naturales (biología, biofísica, bioquímica, etología, etc.), así como con aquéllos aportados por la experiencia histórica y la vida social; para decirlo claramente, una antropología de acuerdo con los avances científicos de nuestro tiempo y que, por lo tanto, debe ofrecer el rasgo de la provisionalidad, la racionalidad de sus afirmaciones y la incesante búsqueda de la globalidad o visión general del sistema humano. Una antropología, así concebida, nunca está acabada pero presenta la solidez de la probabilidad científica mientras que no haya datos que la puedan falsear; ofrece distintos modelos del hombre según la combinación de los datos, los sistemas de valores desde los que se hayan elegido y la potencia mental que se tenga para interpretarlos; tiene la profunda modestia, por lo tanto, de no considerarse exclusiva sino compatible o, al menos, respetuosa con otras imágenes globales (Escámez, 1986, 158).

Lo contrario sería reducir la racionalidad tecnológica a su vertiente instrumental, limitando la acción y su propia racionalidad, con lo cual caeríamos en la denuncia que formulaba el profesor J. Muguerza (1986), en la razón sin esperanza, en la unilateralidad de la razón instrumental sin el apoyo de la racionalidad práctica.

Es cierto, no cabe la menor duda, que la tecnología y su normatividad fijan las claves del éxito de su actuación en la sistemática y racional ordenación de las acciones bajo los criterios de eficacia y controlabilidad de la realidad; no obstante, no es menos cierto que la racionalidad de una acción debe afectar, tanto al proceso (ordenación de las acciones), como a su resultado, el cual debe venir respaldado por los fines y objetivos que se marcan. Desde esta perspectiva, una acción podemos juzgarla racionalmente correcta desde la normatividad propia de la ciencia y la tecnología, y ser irracionalmente incorrecta desde la normatividad prudencial o ética:

En otras épocas se daba por supuesto que el progreso de la razón y de la eticidad estaba regido por un principio que unificaba todas las manifestaciones. El hombre, mediante el ejercicio libre de la razón, caminaba automáticamente hacia la plenitud total como ser humano y ser social. Hoy podemos constatar que no es así. El desarrollo industrial, la tecnificación de la vida, lo que llamamos sociedades altamente evolucionadas, promueven ciertas formas de racionalidad e inhiben otras. Incluso generan irracionalismos inevitables que hacen su aparición constante en la vida cotidiana y en muchos aspectos de la vida personal. Del mismo modo, inducen conductas responsables al tiempo que fomentan la irresponsabilidad y el egoísmo (Rodríguez Neira, 1992, 82). 
La acción tecnológica, en cuanto acción intencional e intencionada, como ya hemos dicho, busca conseguir objetivos de valor. La acción tecnológica, sin embargo, no se desarrolla aséptica y objetivamente, sino que normalmente está mediada y envuelta en creencias, experiencias previas, valores, actitudes, opiniones, prejuicios, sentimientos, es decir, por la red simbólico-cultural del sujeto y del colectivo en el que se desenvuelve (Castillejo, 1987; Elkana, 1983) que condicionan el desarrollo general de la acción. Estas, llamémosle, representaciones previas, o si se quiere, como las llaman los profesores J. García Carrasco y A. García del Dujo, teorias personales ${ }^{11}$, deben ser tenidas en cuenta a la hora de diseñar acciones tecnológicas, pues, a menudo e inconscientemente, son estas teorías las que dirigen verdaderamente nuestras actuaciones, adaptando los procesos a las creencias particulares, impidiendo, con ello, la crítica y la autocrítica, cayendo en actuaciones irracionales, rutinarias, sin verdadero sentido crítico-tecnológico ${ }^{12}$ :

El sujeto perceptor no es un escribiente eficaz que registra pasivamente fragmentos de información. El sujeto perceptor es un intérprete activo, que resuelve ambigüedades, hace suposiciones informadas sobre eventos que no puede observar directamente, y forma inferencias sobre asociaciones y relaciones causales [...] Esbozamos algunas de las "estructuras de conocimiento" aplicadas a la comprensión del mundo. Éstas van desde teorías proposicionales amplias sobre la gente en general hasta representaciones más esquemáticas de objetos, eventos, y actores. Estas estructuras albergan el conocimiento genérico de la persona y sus preconcepciones sobre el mundo, y constituyen la base para una interpretación rápida, coherente, pero ocasionalmente errónea, de la nueva experiencia (Nisbett y Ross, 1980, 17).

El sistema de creencias de los sujetos, la toma de decisiones y la materialización de las acciones, por lo tanto, guardan una estrecha relación que no se puede ni se debe ignorar ${ }^{13}$. Por ello, más que buscar una secuenciación lineal en el desarrollo de

11. "Las teorías personales dirigen y limitan la atención de los sujetos [...] Nuestras teorías personales confeccionan la parte que les corresponde de nuestros sistemas de preferencias, y éstas son las que dirigen y sostienen la atención [...] Nuestros conocimientos, experiencias, actitudes, opiniones, prejuicios, sentimientos... configuran este sistema teórico; es el mediador de nuestras relaciones con el mundo, personaliza los significados de las informaciones que obtenemos del mundo. Lo que hacemos en el mundo depende de lo que creemos sobre él; nuestras percepciones son interpretativas y el código de interpretación del mundo es nuestro sistema de símbolos comunitariamente compartidos y personalmente organizado" (García Carrasco y García del Dujo, 1996, 219).

12. Las ciencias cognitivas han influido enormemente en el estudio de los procesos de toma de decisiones y en el estudio finalístico de las acciones humanas. Ver, por ejemplo, Aparicio y Rodríguez Moneo, 2000; Martín Ortega, 2000.

13. Véase, por ejemplo, la importancia de los valores (sistema de creencias) por el papel que desempeñan en el plano cognitivo, afectivo y conductual. En este sentido Rokeach es uno de los psicólogos sociales que más han profundizado en el tema. Para Rokeach un valor es una creencia duradera donde un modo de conducta o estado último de existencia es personal y socialmente preferible a su opuesto o inverso. Todo valor tiene tres componentes o dimensiones: cognitiva, afectiva y conductual. 
las acciones tecnológicas (conjunto sistemático de acciones lógicamente ordenadas que se aplican siguiendo una mecánica prefijada en función de la coherencia interna del sistema), es preferible atender, sin infravalorar aquéllos, a la importancia y significatividad que los programas de actuación tienen para los agentes implicados (significados que se hacen efectivos en la red simbólica propia de los participantes), la correcta adecuación de los recursos disponibles a los objetivos previstos, y el grado de controlabilidad que se ejerce sobre la realidad (Toribio, 1995). Por otra parte, es preciso matizar que los resultados finales de nuestras acciones son muy importantes, sin embargo, nunca deben minimizar el papel de los resultados parciales, del reajuste de nuestros objetivos, de la readecuación de nuestras decisiones, así como el propio proceso (y posibles subprocesos) de puesta en marcha de nuestras acciones, con todas las variables que van apareciendo, en especial las afectivo-motivacionales (White, 1990; Fullan, 1991; Zimmerman, 1996). Los contextos donde las acciones tecnológicas se desarrollan son altamente complejos, inciertos, cambiantes, dinámicos, formados por un número de variables (controlables y no controlables) y elementos interdependientes e intercambiables de naturaleza dispar: cuantitativos y cualitativos (Castillejo, 1987). Además, esta complejidad e incertidumbre impide en gran medida inferir predictivamente los acontecimientos y los resultados, dándose paso, con ello, más que a la previsión, a la opcionalidad que depende, en gran medida, de las propias características de los contextos en los que nos movemos ${ }^{14}$.

\section{El PLANTEAMIENTO TECNOCIENTÍfiCO EN EL COMPLEJO MUNDO DE LA EDUCACIÓN}

M. Bunge (1985b) entendía las ciencias tecnológicas como aquellas que se incardinaban a la acción práctica, encontrándose entre ellas la Pedagogía. El saber y el saber bacer con finalidades educativas es lo que denominamos saber pedagógico. El conocimiento pedagógico es una forma específica y especializada de saber que incide directamente sobre la práctica educativa, a partir de un sistema pertinente de conocimientos tecnocientíficos que deben ser conocidos (ciencia), aplicados (técnica) y criticados (ética), con la finalidad de incidir eficazmente en los procesos de enseñanza y aprendizaje desarrollados, orientándolos en la dirección deseada. El

El sistema de valores surge como resultado de los cambios en la cultura, en la sociedad y en la experiencia personal, desempeñando funciones tan importantes como la normativa y la motivacional. Para una a proximación sintética al pensamiento de este autor, véase el trabajo de J. Garcés (1988).

14. "El orden es el lugar desde el que se piensa y desde donde opera la racionalidad. Por eso la ciencia (de la modernidad) entiende el orden en razón inversa a la incertidumbre por lo que su cometido es aportar verdades, certezas. El caso de la educación es en este sentido paradigmático; el filosofismo de la pedagogía se deshace en el devenir del siglo xx en aras de la racionalidad y especialización que le aportan las ciencias de la educación. En cambio, en la postmodernidad, o cultura social de las nuevas tecnologías, la certidumbre ha perdido su valor; no interesa tanto la verdad como la satisfacción; de la lógica de la razón se ha pasado a la lógica de la necesidad, de tal manera que ha ido desapareciendo el pensamiento fundacional para dar paso a la opcionalidad [...]" (Colom, 2001, 5). 
éxito de dicho conocimiento se fundamenta en la posibilidad de promover una actitud intelectual transformativa (el profesional como intelectual transformativo) basada en el razonamiento pedagógico científico-tecnológico, que nos permita el tránsito o aproximación desde la realidad fáctica a la realidad deseada: racionalidad epistémica, racionalidad tecnológica y racionalidad prudencial. Este conocimiento debe ser, con independencia del paradigma en el que nos coloquemos como educadores, eminentemente tecnológico. El saber pedagógico es un saber para intervenir, para desarrollar acciones educativas dirigidas a la optimización del sujeto y su realidad. Saber tecnológico que, como hemos visto, halla subsidiariedad en la ciencia y en la ética.

Como sabemos, tres son las formas paradigmáticas con las cuales se interpreta la realidad: tecnológica, crítica y bermenéutica. Personalmente esta clasificación no me satisface porque tiende a confundir y enmascarar la realidad. Parece que el paradigma crítico-hermenéutico fuera ajeno a los planteamientos tecnológicos, cuando en realidad no lo es, pues, desde el momento en que quiere intervenir para mejorar la realidad debe hacerlo desde posicionamientos tecnológicos (saber para hacer), de lo contrario caería en la irracionalidad, en el actuar por actuar, sin ningún tipo de significado ni racionalidad (Bernabeu y Colom, 1997a). En este sentido, es preferible hablar, no de paradigma tecnológico, sino de paradigma conductista (conducción de un conjunto ordenado y coherente de acciones prepensadas, ligadas al contexto y a la significación de los agentes participantes, para la mejora intencional de la realidad factual). Pienso que paradigma conductista, despojado de las connotaciones negativas de las primeras teorías asociacionistas (estímulo-respuesta), responde mejor a la cuestión, si bien, todo se reduce, en última instancia, más a un problema de significados que no de significantes o, dicho de otra manera, de actitudes. Aclarado este punto, hemos de decir que la práctica pedagógica es un sistema de acciones claramente intencionales que busca lograr objetivos y finalidades educativas explicitadas (García Carrasco, 1986). Las racionalidades del saber pedagógico, en esencia, no se diferencian de las racionalidades del saber en general, pues la racionalidad de una acción pedagógica tecnológicamente concebida implica lo epistémico (ciencia), lo instrumental (técnica) y lo práctico (ética). La racionalidad de una acción pedagógica debe atender, de partida, los fines educativos (intencionalidad y propositividad optimizante o valorizante, en el sentido de hacer más valioso al ser humano), sin embargo, de poco o nada nos sirven los fines o grandes principios orientadores si no recurrimos a su operativización tecnológica que, a su vez, como ya hemos dicho, se sostiene en la ciencia. Dicho en otras palabras, la racionalidad de una acción educativa supone tratar la acción en su totalidad o globalidad sistémica.

Los fines están ligados a los valores humanos y al proceso humanizador en el desarrollo de la personalidad moral. La racionalidad tecnológica debe atender, no sólo a los medios, sino también a los fines. Este hecho, en ocasiones, a veces más de lo deseado, es olvidado, atendiéndose sólo a los medios, descuidándose, subordinándose o relegándose a un segundo plano, por inútiles o intrascendentes, 
las cuestiones fundamentales que afectan a los fines que, precisamente, dotan de sentido y significado a la acción y a los propios medios que se utilizan: «[...] la condición necesaria a toda actividad pedagógica válida es el plan de la obra, por lo que se impone tratar primero los problemas axiológicos. Se quiere decir con ello que la metodología, la técnica y la experimentación no son nada si no contienen una finalidad, y esta finalidad vendrá determinada por los valores. [... En este sentido] la metodología (todo aquello que haga alusión a medios) está supeditada y es posterior a la axiología” (Núñez Cubero, 1986, 56).

El saber práctico y sus racionalidades, por lo tanto, implican la producción de un saber actuar operativo eficaz, al tiempo que un conocimiento crítico-reflexivo capaz de discriminar lo educativo de lo no educativo, lo moral de lo no moral; sólo así es posible dotar al sistema educativo (sistema de acciones educativas) de un significado humanizador y moralizante ${ }^{15}$. Cualquier pretensión reduccionista o absolutista de alguna o algunas de las racionalidades que integran nuestro especial conocimiento distorsionaría y limitaría el propio ejercicio de la racionalidad ${ }^{16}$. La experiencia, sin embargo, nos dice que la racionalidad tecnológica suele identificarse o reducirse a la racionalidad instrumental. La incidencia del positivismo del primer tercio del siglo xx y su posterior desarrollo en las ciencias humanas y sociales (y con ellas la Pedagogía ${ }^{17}$ en la búsqueda de una objetiva cientificidad para sus conocimientos, hizo que se rompiera todo nexo o relación con la filosofía, adoptando y adaptando, para ello, la metodología propia de las ciencias naturales (Dilthey, 1978; Rickert, 1965), lo cual incidió negativamente en su objeto de estudio, en el bombre y su mundo (mundo de la vida) ${ }^{18}$, el cual, al serle aplicado el formalismo lógico-experimental del positivismo, quedó altamente reducido y codificado en su significación, potenciando la razón instrumental en detrimento de la razón práctica. Sobre los años sesenta y setenta del siglo xx la incidencia de los presupuestos epistemológicos y metodológicos de la Teoría General de Sistemas $^{19}$ y de la primera cibernética (Wiener, 1969, 1971, 1986; Ashby, 1965, 1972) seguirá

15. En este sentido, es interesante ver los trabajos contenidos en la obra coordinada por J. C. Mèlich (2000).

16. G. Vázquez (1997), en consonancia con lo dicho, señala la importancia de diferenciar entre virtud y técnica, y de situar la primera en el centro de la preocupación y ocupación pedagógica. En un sentido parecido se pronuncia la profesora A. Cortina (1993).

17. Baste recordar la escisión que se produce en el primer tercio del siglo xx, a nivel metodológico, dando lugar a una pedagogía de corte herbartiano que pasará a llamarse racional (F. Paulsen) y otra de corte positivista denominada experimental (W. A. Lay y E. Meuman).

18. La teoría sociológica contemporánea ha procurado analizar la realidad social desde dos puntos de vista radicalmente opuestos: por una parte, el sistema (Gesellschaft); y, por otra, el mundo de la vida (Gemeinsschaft). El sistema y el mundo de la vida son dos categorías sociológicas básicas que han dado lugar a dos modos de ver lo social. Véanse Habermas, 1985, 1996; Gómez-Heras, 1989; Izuzquiza, 1990; Jiménez, 1998; García-Baró, 1999; Luhmann, 1997.

19. En oposición a las teorías personalistas y a las últimas propuestas de la fenomenología y de la teoría de los valores, a finales de los años sesenta del siglo xx, surge la reacción materialista (las nuevas teorías de la posmodernidad), que se acercan a los presupuestos epistemológicos de las ciencias de 
potenciando la instrumentalidad de la razón tecnológica, poniendo especial atención en la aplicación práctica de los principios sistémicos para la resolución de problemas sociales y educativos a través de la nueva epistemología y metodología sistémica.

La racionalidad tecnológica que estructura el conocimiento pedagógico debe implicar, no sólo una racionalidad instrumental (coherencia de medios y fines), sino también un conocimiento de los grandes principios, fines y objetivos del sistema y de sus acciones, además de un conocimiento científico del sistema, situación, contexto o realidad sobre la que se interviene. De esta manera la eficacia de una acción pedagógica posibilita y permite un análisis de la viabilidad prudencial o ético-moral de los valores que intervienen, y que no pueden ser ignorados en la toma de decisiones pedagógicas acerca de la metodología de actuación ${ }^{20}$. La racionalidad práctica nos asegura que las acciones desarrolladas se orientan en la dirección deseada, dotando a nuestras actuaciones de consistencia y coherencia ético-moral. Una vez que dotamios de coherencia y consistencia prudencial a nuestro sistema, el saber pedagógico debe asegurar también un conocimiento tecnológico sistemáticamente planificado que nos permita normativizar y orientar, eficaz y eficientemente, el modo como conseguir los objetivos que se han propuesto. Lo verdaderamente problemático de la pedagogía tecnológica consiste, sobre todo, en los aspectos derivados de la razón prudencial, es decir, de la normatividad ético-axiológica, pues en el marco de la pluralidad propia del contexto de la sociedad civil democrática éstos son complejos y, en ocasiones, contrapuestos. El problema consistirá, por lo tanto, en el tipo de procedimientos que entran en juego al aplicar los criterios de la razón instrumental: eficacia/eficiencia y control de la realidad que se consideran más adecuados para la resolución de problemas y transformación de la realidad, sin ignorar los presupuestos que se desprenden de la racionalidad prudencial que será, precisamente, la guía axiológica de nuestras actuaciones.

La ciencia actúa, como ya hemos dicho, por medio de la teoría (forma de conocimiento asociada al saber científico), en contraposición a la tecnología que se orienta y estructura alrededor de los modelos. La cientificidad del conocimiento propio del saber teórico de la pedagogía precisa, indefectiblemente, de la racionalidad científica y, hasta cierto punto, un distanciamiento del saber filosófico que,

la naturaleza (epistemología compartida): teorías sistémico-cibernéticas. Sobre la década de los ochenta, profundizando en el saber sistémico, van a surgir nuevos planteamientos como la teoría de la complejidad, la teoría del caos, los modelos ecológicos etc. La nota común a todas ellas es reconvertir la germánica Pedagogía de las creencias y las ideologías en una propuesta positivista de Pedagogía de la racionalidad factual. Véanse, entre otros, los siguientes trabajos: Finkielkraut, 1987; Derrida, 2001; Foucault, 1990; Lipovetski, 1991; Vattimo y Rovatti, 1992; Lyotard, 1989; Bertalanffy, 1981; Bertalanffy y Laszlo, 1981; Luhmann, 1993; Toffler, 1990; Cohn, 1995; Ferris, 1998; Moles, 1995.

20. En este sentido se pronuncia M. Á. Quintanilla (1989), para quien la racionalidad teleológica de toda tecnología debe evaluarse por el criterio del grado de control sobre los procesos que trata de modificar, por lo que la racionalidad tecnológica implica responder a los criterios de eficacia, utilidad e innovación. 
no obstante, debe preservarse para la orientación de los grandes principios y fines que dirigen los procesos educacionales. Lo cierto, sin embargo, es que las fronteras entre la ciencia y la filosofía no están claramente delimitadas (Losse, 1985), y si la concepción clásica de la cientificidad del saber pedagógico demandaba una radical separación del subjetivo mundo de los valores, a partir de la década de los ochenta del siglo xx se inicia el andamiaje para la construcción de una nueva epistemología que incluye, dentro de los presupuestos de lo caótico, complejo e incierto propio del contexto en el que se mueven nuestros conocimientos, el mundo de los valores, las creencias, las concepciones, las ideologías, etc. Las ciencias naturales ya están participando en la actualidad de un mismo enfoque o paradigma, el mundo de lo complejo (Briggs y Peat, 1989), y las ciencias humanas y sociales parecen orientarse también en esta dirección (Lewin, 1995; Vilar, 1997; Domínguez, 1998; Colom, 2001; Solana, 2001). En este sentido, la cibernética de segundo orden (nueva teoría general de sistemas o teorías de los sistemas observantes) ${ }^{21}$, la nueva teoría de la información y de la comunicación, y las ciencias cognitivas inspiradas en la teoría del caos, pueden resultar un camino válido para llegar a una vía epistemológica compartida de gran valor, tendente a la superación de la clásica clasificación de los saberes, así como de la también clásica separación del mundo de la vida y el de los sistemas, sin que por ello peligre la cientificidad (rigor y objetividad) de los constructos elaborados en el seno de las ciencias humanas y sociales y, por supuesto, en el de las ciencias de la educación.

En este sentido, Y. Elkana (1983) nos dice que las fuentes del conocimiento, y con ellas el rigor y la objetividad, dependen de las imágenes del conocimiento, las cuales varían de una cultura a otra, dependiendo del contexto (variables espacio/tiempo). Ni la ciencia ni ninguna otra disciplina, sigue diciéndonos, se basa sólo sobre una fuente última de conocimiento, por lo cual la autoridad no se acepta como prueba definitiva. Los métodos son imágenes cognitivas, son sinónimos de las jerarquías aceptadas de las fuentes contextuales. Puesto que no existe ninguna certidumbre intercultural, y ya que nuestras guías epistemológicas son las imágenes cognitivas socialmente determinadas, debemos ser realistas dentro del marco conceptual que hallamos escogido y respecto al tipo de conocimiento que buscamos. Podemos pensar, simultáneamente, en dos niveles diferentes y estar sinceramente convencidos de dos tipos de verdades sin reducirlas al mismo nivel. Todo lo que nos parece interesante, bello, científico, verdadero, está determinado

21. La nueva teoría general de sistemas o teoría de los sistemas observantes o de los sistemas fuera del estado de equilibrio tienen sus origenes en la teoría general de los sistemas de Bertalanffy, la cibernética de Wiener y la teoría de la información de Shannon. Los conceptos basicos y las teorías fueron desarrollados en diversos dominios de las ciencias naturales y sociales como tambien en la filosofía. Estos conceptos alcanzaron su madurez con la termodinámica del no equilibrio de Prigogine y los nuevos avances en la construcción de modelos matemáticos del caos y la transformación en los sistemas dinámicos. Estas ciencias nos dan una visión nueva de la naturaleza de la realidad. El hombre y la sociedad no son extraños, sino que forman parte de la gran cadena de la evolución. Estas ciencias describen las características dinámicas de esta evolución y sus principales etapas. 
por nuestras imágenes de conocimiento que se encuentran condicionadas de manera muy específica dentro de los contextos socio temporales dados. Epistemológicamente hablando, según Elkana, deberían tenerse en cuenta dos aspectos: por un lado, la imposibilidad de ordenar objetivamente marcos conceptuales según su grado de racionalidad o de aproximación a una verdad independiente de un contexto; y, por otro, la necesidad de crear una epistemología integral o compartida. El rigor científico consistiría en adoptar aquel procedimiento particular por el que se logra explicar, describir e interpretar un fenómeno en base a hipótesis de trabajo científicamente justificadas y fundamentadas ${ }^{22}$. En el marco de las ciencias humanas y sociales y, en particular, en el de las ciencias de la educación, el rigor científico se puede obtener tomando diversos criterios, siempre que éstos sean consecuentes con los presupuestos teóricos y con las metodología con las que trabajamos. Así pues, podemos hablar de tantas objetividades, de tantos niveles de realidad (imágenes cognitivas socialmente determinadas) sobre las que se estructura el conocimiento científico, como de paradigmas, modelos o enfoques científicos existentes.

Podemos decir, ya para concluir, que la objetividad definida por el positivismo científico minimizó, hasta diluirla, la subjetividad del sujeto. Así, por ejemplo, en el estructuralismo y en el primer pensamiento sistémico, la epistemología del sujeto, la categoría antropológica humana, se vio superada y sustituida por el sistema (Luhmann, 1990; Bertalanffy, 1981) y por la estructura (Foucault, 1971, 1987, 1990). El rigor científico positivista procedía a través de la neutralización de las propiedades del observador (un observador objetivamente incardinado en su papel observante, libre de ataduras subjetivas). Hoy, como hemos dicho, la objetividad científica consiste en un peculiar sistema de representación que no es ni puede ser ajeno al sistema sociocultural en el que se mueve, pues la objetividad, como la ciencia, es una construcción histórico-cultural más, no la única ${ }^{23}$. La nueva perspectiva sistémico-constructivista nos advierte que la objetividad participa, necesariamente, de la subjetividad, pues no hay un observador en estado puro ya que éste es inseparable de lo que observa. La objetividad de la realidad fáctica implica, y complementa, la subjetividad de quien la observa, pues observador, fenómeno observado y proceso de observación configuran una totalidad sistémica indivisible (Maturana y Varela, 1999).

22. W. V. Quine $(1992,1998)$ nos dice que solamente tiene sentido aplicar la palabra verdadero a una frase concebida según los términos de una teoría dada y en vista de esta teoría con su realidad postulada.

23. En este sentido, G. Bateson (1983) plantea 16 presupuestos básicos que todos los espíritus deben compartir: la ciencia nunca prueba nada; el mapa no es el territorio y el nombre no es la cosa nombrada; no hay experiencia objetiva; los procesos de formación de imágenes son inconscientes; la división del universo percibido en partes y totalidades es conveniente y puede ser necesaria, pero ninguna necesidad determina de qué modo debe practicársela; las consecuencias divergentes son impredecibles; las consecuencias convergentes son predecibles; nada puede provenir de la nada; la cantidad no determina la pauta; en biología no hay valores monótonos; a veces lo pequeño es hermoso; la lógica es un modelo deficiente de la causa y el efecto; la causalidad no opera hacia atrás; por lo común el lenguaje sólo destaca uno de los aspectos de una interacción cualquiera; las palabras estabilidad y cambio describen una partes de nuestras descripciones. 


\section{BiBLIOGRAFÍA}

AGAZZI, E., (1996) El bien, el mal y la ciencia: las dimensiones éticas de la empresa científico-tecnológica. Madrid, Tecnos.

ÁLVAREZ CASTILLO, J. L. (2001) Posibilidades y límites político-educativos de las nuevas tecnologías de la información y la comunicación, Revista española de pedagogía, $\mathrm{n}^{\mathrm{O}} 218$, 85-104.

APARICIO, J. J. y RODRÍGUEZ MONEO, M. a (2000) Los estudios sobre el cambio conceptual y las aportaciones de la Psicología del aprendizaje, Tarbiya. Revista de investigación e innovación educativa, no 26, 13-30.

ARANGUREN, J. L. (1973) Sobre ecología y tecnología, Revista de Ciencias Sociales, nº 1, 52 58.

- (1982) La ética en relación con la ciencia y la tecnología, Escuela Técnica Superior de Ingenieros Industriales de Terrasa, no 16, 8-13.

- (1983) Propuestas morales. Madrid, Tecnos.

ASHBY, W. R. (1965) Proyecto para un cerebro: El origen del comportamiento adaptativo. Madrid, Tecnos.

- (1972) Introducción a la cibernética. Buenos Aires, Editorial Nueva Visión.

ASTOLFI, J. P. (1999) El "error, un medio para enseñar: Gaston Bachelard, Jean Piaget. Sevilla, Díada.

ATLAN, H. (1991) Con razón y sin ella. Barcelona, Tusquets.

AYUSTE, A. y otros (1999) Planteamientos de la Pedagogía Crítica. Comunicar y transformar. Barcelona, Graó.

BALANDIER, G. (1996) El desorden y la teoría del caos y las ciencias sociales. Elogio de la fecundidad del movimiento. Barcelona, Gedisa.

BALZER, W. (1997) Teorias empíricas: modelos, estructuras y ejemplos. Los elementos fundamentales de la Teoría Contemporánea de la Ciencia. Madrid, Alianza Editorial.

BATESON, G. (1993) Espiritu y naturaleza. Buenos Aires, Amorrortu.

BERNABEU, J. L. y COLOM, A. J. (1997a) El paradigma crítico-hermenéutico y el paradigma tecnológico, en COLOM, A. J. (coord.). Teorias e instituciones contemporáneas de la educación. Barcelona, Ariel, 157-169.

BERTALANFFY, L. von (1981) Teoria general de los sistemas: fundamentos, desarrollo, aplicaciones. México, Fondo de Cultura Económica.

- y LASZLO, E. (1981) Hacia una filosofía de sistemas. Valencia, Teorema.

BOHM, D. (1992) La totalidad y el orden implicado. Barcelona, Kairós.

BRIGGS, J. y PEAT, D. (1999) Las siete leyes del caos: las ventajas de una vida caótica. Barcelona, Grijalbo.

BUNGE, M. (1985a) Seudociencia e ideologia. Madrid, Alianza.

- (1985b) Epistemología. Curso de actualización. Barcelona, Ariel.

CASTILlejO, J. L. (1987) Pedagogía tecnológica. Barcelona, CEAC.

COHN, H. (1995) El cosmos, el caos y el mundo venidero. Barcelona, Crítica.

COLOM, A. J. (1986) Pensamiento tecnológico y teoría de la educación, en CASTILlEJO, J. L. y otros. Tecnología y Educación. Barcelona, CEAC, 13-30.

- (1997a) La teoría de la educación: contexto actual de los estudios pedagógicos, en COLOM, A. J. (coord.). Teorías e instituciones contemporáneas de la educación. Barcelona, Ariel, 145-155. 
- (1997b) La regionalización de la educación como tecnología cognitiva virtual. Teoría de la Educación. Revista Interuniversitaria, no 9, 7-19.

- (2001) Teoría del caos y educación (Acerca de la reconceptualización del saber educativo). Revista española de pedagogia, $\mathrm{n}^{\circ}$ 218, 5-24.

COPELAND, J. (1996) Inteligencia artificial. Madrid, Alianza.

CORTINA, A. (1993) Ética aplicada y democracia radical. Madrid, Tecnos.

CHALMERS, A. F. (1989) ¿Qué es una cosa llamada ciencia? Madrid, Siglo XXI Editores.

DERRIDA, J. (2001) La deconstrucción en las fronteras de la filosofía: la retirada de la metáfora. Barcelona, Paidós Ibérica.

DEWEY, J. (1995) Democracia y Educación. Madrid, Morata.

DILTHEY, W. (1978) Introducción a las Ciencias del Espiritu. México, Fondo de Cultura Económica.

DOMÍNGUEZ, J. L. (1998) La gran metáfora: una particular aproximación a la complejidad de los sistemas. Madrid, Vulcano.

ELKANA, Y. (1983) La ciencia como sistema cultural: una aproximación antropológica. Boletín de la Sociedad Colombiana de Epistemologia, vol. III ( $n^{\text {os }} 10-11$ ), 65-80.

ESCÁMEZ, J. (1986) Los valores en la pedagogía de la intervención, en CASTILLEJO, J. L. y otros. Tecnología y Educación. Barcelona, CEAC, 155-173.

FERNÁNDEZ SÁEZ, I. (1993) Inconmensurabilidad y racionalidad cientîfica. Madrid, Universidad Complutense.

FERRIS, T. (1998) Informe sobre el universo. Barcelona, Crítica.

FEYERABEND, P. K. (1999) Tratado contra el método: esquema de una teoría anarquista del conocimiento. Barcelona, Altaya.

FINKIELKRAUT, A. (1987) La derrota del pensamiento. Barcelona, Anagrama.

FOUCAULT, M. (1971) Las palabras y las cosas. Madrid, Siglo XXI.

- (1987) El orden del discurso. Barcelona, Tusquets.

- (1990) Tecnologias del yo. Barcelona, Paidós/ICE de UAB.

FOX, A. y FOX, B. (1995) Más allá del pensamiento positivo: del pensamiento a la acción. Madrid, Los Libros del Comienzo.

FULLAN, M. (1991) The new meaning of educational change. London, Cassell.

GARCÉS, J. (1988) Valores bumanos. Principales concepciones teóricas. Valencia, Nau Llibres.

GARCÍA-BARÓ, M. (1999) Vida y mundo: la práctica de la fenomenología. Madrid, Trotta.

GARCÍA CARRASCO, J. (1986) Reflexión sobre la intervención pedagógica a propósito de un estudio de Talcott Parson, en CASTILlEJO, J. L. y otros. Tecnología y Educación. Barcelona, CEAC, 125-153.

- y GARCía DEL DUJO, A. (1996) Teoria de la Educación I. Educación y acción pedagógica. Salamanca, Universidad de Salamanca.

GERGEN, K. J. (1996) Realidades y relaciones: aproximaciones a la construcción social. Barcelona, Paidós Ibérica.

GIMENO, J. (1981) Teoria de la enseñanza y desarrollo del curriculum. Madrid, Anaya.

GIORDAN, A. y VECCHI, G. de. (1988) Los Origenes del Saber: De las Concepciones Personales a los Conceptos Científicos. Sevilla, Diada Editores.

GLEICK, J. (1988) Caos: la creación de una ciencia. Barcelona, Seix-Barral.

GÓMEZ-HERAS, J. M. a (1989) El apriori del mundo de la vida: fundamentación fenomenológica de una ética de la ciencia y de la técnica. Barcelona, Anthropos.

HABERMAS, J. (1985) La modernidad, un proyecto incompleto. Barcelona, Kairós.

- (1996) Conciencia moral y acción comunicativa. Barcelona, Península. 
HAYMAN, J. L. (1981) Investigación y educación. Barcelona, Paidós.

HORGAN, J. (1998) El fin de la ciencia, los limites del conocimiento y el declive de la era científica. Barcelona, Paidós.

HOTTOIS, G. (1999) Historia de la Filosofia del Renacimiento a la Posmodernidad. Madrid, Cátedra.

IZUZQUIZA, I. (1990) La sociedad sin hombres. Niklas Lubmann o la teoría como escándalo. Barcelona, Anthropos.

JIMÉNEZ, D. (1998) La reformulación de la critica de la cosificación por J. Habermas: la colonización del mundo de la vida. Cádiz, Universidad de Cádiz.

KAUFMAN, R. A. (1977) Planificación de sistemas educativos. México, Trillas.

KERLINGER, F. N. (1975) Investigación del comportamiento. México, Interamericana.

KUHN, T. S. (1981) La estructura de las revoluciones científicas. Madrid, Fondo de Cultura Económica.

- (1995) ¿Qué son las revoluciones científicas? Barcelona, Altaya.

LAKATOS, I. (1978) Pruebas y refutaciones: la lógica del descubrimiento matemático. Madrid, Alianza.

- (1981) La crítica y la metodología de programas científicos de investigación. Valencia, Revista Teorema.

- y MUSGRAVE, A. (eds.) (1970) Criticism and the growth of knowledge. Cambridge, University Press.

LEWIN, R. (1995) Complejidad: el caos como generador del orden. Barcelona, Tusquets.

LIPOVETSKI, G. (1991) El imperio de lo efimero. Barcelona, Anagrama.

LIZ, M. (1995) Conocer y actuar a través de la tecnología, en BRONCANO, F. (ed.). Nuevas meditaciones sobre la técnica. Madrid, Trotta, 23-51.

LÓPEZ-BARAJAS, E. (1984) Didácticas: metodología de la investigación. Madrid, UNED.

LOSSE, J. (1985) Introducción bistórica a la filosofía de la ciencia. Madrid, Alianza Editorial.

LUHMANN, N. (1990) Sociedad y sistema: la ambición de la teoría. Barcelona, Paidós.

- (1993) Teoría y práctica en el Estado del Bienestar. Madrid, Alianza.

- (1997) Observaciones de la modernidad: racionalidad y contingencia en la sociedad moderna. Barcelona, Paidós.

LYOTARD, J. F. (1989) La condición postmoderna. Madrid, Cátedra.

MARTÍN ORTEGA, E. (2000) Puede ayudar la teoría del cambio conceptual a los docentes, Tarbiya. Revista de investigación e innovación educativa, $\mathrm{n}^{\circ}$ 26, 31-50.

MARTÍNEZ MIGUÉLEZ, M. (1993) El paradigma emergente: hacia una nueva teoría de la racionalidad cientifica. Barcelona, Gedisa.

MATURANA, H. y VARELA, F. (1999) El árbol del conocimiento: las bases biológicas del conocimiento bumano. Madrid, Debate.

MEDINA DOMÍNGUEZ, J. (coord.) (1999) Historia de la ciencia y de la técnica. Madrid, Centro de profesores y Recursos de Aranjuez.

MÈLICH, J. C. y otros (coords.) (2000) La veu de l'altre: reflexions i experiències per educar en valors ètics. Barcelona, ICE de la Universitat Autònoma de Barcelona.

MIRÓ, J. (ed.) (2001) VII Jornadas de Enseñanza Universitaria de la Informática. Palma de Mallorca, Universitat de les Illes Balears.

MOLES, H. (1995) Les sciences de l'imprecis. Paris, Du Seuil.

MORENO CÁDIZ, L. J. (1989) La última argucia de la razón práctica. Cádiz, Caja de Ahorros de Cádiz.

MORIN, E. (1995) Introducción al pensamiento complejo. Barcelona, Gedisa. 
MUGUERZA, J. (1986) La razón sin esperanza. Siete trabajos y un problema de ética. Madrid, Taurus.

NEWTON-SMITH, W. H. (2000) La racionalidad de la ciencia. Barcelona, Paidós Ibérica.

NISBETT, R. y ROSS, L. (1980) Human inference: Strategies and shortcomings of social judgement. Englewood Cliffs, NJ, Prentice-Hall.

NOVAK, J. D. (1993) Human constructivism: a unification of psicological and epistemological phenomena in meaning making. Internacional Journal of Personal Construct Psychology, no 2, 167-194.

NÚÑEZ CUBERO, L. (1986) La educación construible. Bases para una teoría dinámica de la educación. Sevilla, Publicaciones de la Universidad de Sevilla.

PAGELS, H. R. (1991) Los sueños de la razón: el ordenadory los nuevos horizontes de las ciencias de la complejidad. Barcelona, Gedisa.

PEÑALVER, C. (1987) Las explicaciones sistémicas: una aproximación a la pedagogía sistémica, en CASTILlejO, J. L. y COLOM, A. J. (eds.). Pedagogía sistémica. Barcelona, CEAC, 52-54

PÉREZ RANSANZ, A. R. (1999) Khun y el cambio científico. México, Fondo de Cultura Económica.

PESTRE, D. (1995) Pour une histoire sociale et culturelle des sciences. Nouvelles définitions, nouveaux objets, nouvelles pratiques. Annales d'Histoire et Sociologie des sciences, $\mathrm{n}^{-} \mathrm{3}$, 487-522.

POPPER, K. (1973) La lógica de la investigación científica. Madrid, Tecnos.

- (1983) Conjeturas y refutaciones: el desarrollo del conocimiento científico. Barcelona, Paidós.

- (1988) Conocimiento objetivo: un enfoque evolucionista. Madrid, Tecnos.

PORTA, M. (1983) El positivismo lógico: el Círculo de Viena. Barcelona, Montesinos.

PUIG, J. M. (1986) Teoría de la educación. Una aproximación sistémico-cibernética. Barcelona, PPU.

QUINE, W. V. (1992) La búsqueda de la verdad. Barcelona, Crítica.

- (1998) Del estímulo a la ciencia. Barcelona, Ariel.

QUINTANILLA, M. Á. (1978) El estatuto epistemológico de las Ciencias de la Educación, en ESCOLANO, A. y otros. Epistemología y Educación. Salamanca, Sígueme.

- (1989) Las virtudes de la racionalidad instrumental, Anthropos. Revista de Documentación Científica de la Cultura, nos 94/95, 95 a 98.

RESCHER, N. (1999) Razón y valores en la era científico-tecnológica. Barcelona, Paidós Ibérica, Instituto de Ciencias de la Educación de la Universidad Autónoma de Barcelona.

RICKERT, H. (1965) Ciencia cultural y Ciencia Natural. Madrid, Espasa-Calpe.

RODRÍGUEZ NEIRA, T. (1992) Algunas formas de la racionalidad, Teoría de la Educación, vol. IV, 73-89.

ROMERO, C. (2000) El conocimiento del tiempo educativo. Barcelona, Laertes

RUBIO CARRACEDO, J. (1984) Positivismo, bermenéutica y teoría crítica en las ciencias sociales. Barcelona, Humanistas.

SANVICENS, A. (1986) Teoría cibernética de la acción, en CASTILLEJO, J. L. y otros. Tecnología y Educación. Barcelona, CEAC, 31-51.

SOLANA, J. L. (2001) Antropología y complejidad bumana: la antropología compleja de Edgar Morin. Granada, Comares.

SOLÍS SANTOS, C. (1994) Razones e intereses: la historia de la ciencia después de Kubn. Barcelona, Paidós Ibérica. 
SUPPE, F. (1990) La estructura de las teorias cientificas. Madrid, UNED.

TOFFLER, A. (1990) El "Shock" del futuro. Barcelona, Plaza y Janés Editores

- (1991) El cambio del poder. Barcelona, Plaza y Janés Editores.

TORIBIO, J. (1995) Semántica de las reglas tecnológicas: eficiencia y control en la organización y planificación de los sistemas tecnológicos, en BRONCANO, F. (ed.). Nuevas meditaciones sobre la técnica. Madrid, Trotta, 121-137.

UNESCO (1999) Los siete saberes necesarios para la educación del futuro. Elaborado para la Unesco por Edgar Morin como contribución a la reflexión internacional sobre "Cómo educar para un futuro sosteniblew. París, Unesco.

VATTIMO, G. y ROVATTI, P. A. (1992) La secularización de la filosofía. Hermenéutica y posmodernidad. Barcelona, Gedisa.

VÁZQUEZ GÓMEZ, G. (1997) Valores y "escuela pública” en la sociedad civil, en ORTEGA RUIZ, P. (coord.). Educación Moral. Murcia, CajaMurcia, 109-125.

VILAR, S. (1997) La nueva racionalidad: comprender la complejidad con métodos transdisciplinarios. Barcelona, Kairós.

WAGENSBERG, J. (1989) Ideas sobre la complejidad del mundo. Barcelona, Tusquets.

WHITE, D. J. (1990) Teoría de la decisión. Madrid, Alianza.

WIENER, N. (1969) Cibernética y Sociedad. Buenos Aires, Sudamericana.

- (1971) Cibernética. Madrid, Guadiana.

- (1986) Cibernètica o Control i comunicació en l'animal i la mäquina. Barcelona, Edicions Científiques Catalanes.

ZAMORA, J. A. (2001) La cultura como industria de consumo. Su critica en la Escuela de Francfort. Barcelona, Cristianisme i Justícia.

ZIMMERMAN, B. J. y otros (1996) Developing self-regulated leaners. Beyond achievement to self-efficacy. Washington, DC, APA. 